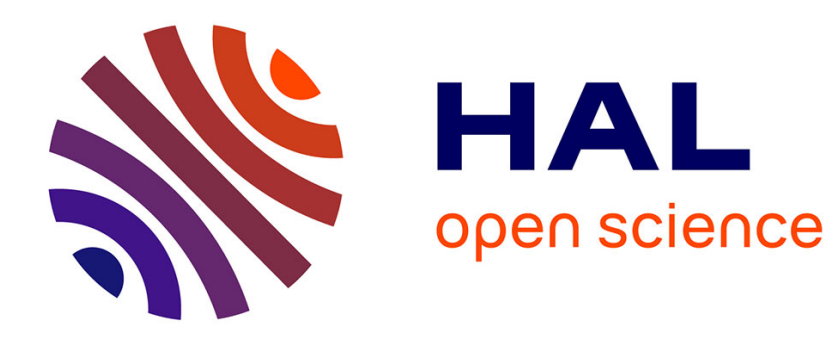

\title{
Remarques sur les contraintes résiduelles
}

Pierre Bérest, Gérard Vouille

\section{- To cite this version:}

Pierre Bérest, Gérard Vouille. Remarques sur les contraintes résiduelles. Comptes Rendus Mécanique, 2003, 331 (6), pp.455-460. 10.1016/S1631-0721(03)00090-1 . hal-00111381

\section{HAL Id: hal-00111381 \\ https://hal.science/hal-00111381}

Submitted on 2 Apr 2018

HAL is a multi-disciplinary open access archive for the deposit and dissemination of scientific research documents, whether they are published or not. The documents may come from teaching and research institutions in France or abroad, or from public or private research centers.
L'archive ouverte pluridisciplinaire HAL, est destinée au dépôt et à la diffusion de documents scientifiques de niveau recherche, publiés ou non, émanant des établissements d'enseignement et de recherche français ou étrangers, des laboratoires publics ou privés. 


\title{
Remarques sur les contraintes résiduelles
}

\author{
Pierre Bérest ${ }^{\mathrm{a}, *}$, Gérard Vouille ${ }^{\mathrm{b}}$ \\ ${ }^{a}$ Groupement d'intérêt public "groupement pour l'étude des structures souterraines de stockage»(GIP-G.3S) et laboratoire de mécanique \\ des solides (LMS), École polytechnique, 91128 Palaiseau cedex, France \\ ${ }^{\mathrm{b}}$ Centre de géotechnique et exploitation du sous-sol (CGES), École nationale supérieure des mines de Paris, 35, rue Saint-Honoré, \\ 77305 Fontainebleau cedex, France
}

\begin{abstract}
Résumé
Les contraintes résiduelles observées dans les solides peuvent résulter de déformations initiales non compatibles. Elles sont alors déterminées, en général, par le tenseur d'incompatibilité du tenseur des déformations initiales. Toutefois, réciproquement, la nullité du tenseur d'incompatibilité ne suffit pas pour conclure à l'absence de contraintes résiduelles, dans le cas où le solide est non simplement connexe, ou présente des surfaces de discontinuité. Plusieurs exemples sont présentés.
\end{abstract}

Mots-clés : Milieux Continus ; Contraintes résiduelles

\section{Abstract}

Some comments on residual stresses. Residual stresses which are currently observed in solid bodies can result from noncompatible initial strains. Theses stresses can then be determined, in general, from the incompatibility tensor associated to the initial strains tensor. However, even if the incompatibility tensor is zero, residual stresses may exist, when the solid is not simply-connected or when discontinuity surfaces are present. Several examples are provided.

Keywords: Continuum mechanics; Residual stresses

\section{Abridged English version}

Residual stresses have two distinct origins: either the initial strains are incompatible (i.e., the strain field $\underline{\varepsilon}^{0}=\underline{\underline{\varepsilon}}^{0}(\underline{x})$, obtained when setting stresses equal to zero in the material constitutive law, cannot be derived from a

\footnotetext{
* Auteur correspondant.

Adresse e-mail : berest@1ms.polytechnique.fr (P. Bérest).
} 
displacement field, $\underline{\underline{\varepsilon}}^{0}=\underline{\underline{\varepsilon}}^{0}\left\{\underline{\xi}^{0}\right\}$, according to (1), because the incompatibility tensor $\underline{\underline{E}}^{0}=\underline{\underline{E}}\left\{\underline{\varepsilon}^{0}\right\}$, as defined by (2), does not vanish to zero); or the considered body is the assembly of several pieces which have been pre-deformed to make their contact surfaces exactly fit together.

When computing residual stresses in a body, it is generally assumed that some loaded state is known; then the body is unloaded; during the unloading, the constitutive behavior is assumed to be expressed by (3), where $\underline{\varepsilon}^{0}$ is the initial strain, $\underline{\sigma}$ is the stress tensor, $\underline{\xi}$ is the displacement from some reference state. The residual $\overline{\bar{s}}$ state of stress is given $\overline{\overline{b y}}$ (4) where the last condition of (4) stipulates that $\underline{\xi}$ must be continuous and must satisfy the closure conditions when the considered body is not simply connected. Simple algebra yields to the system (5) that highlights the role played by the incompatibility tensor of the initial strain field, or $\underline{\underline{E}}^{0}=\underline{\underline{E}}\left\{\underline{\varepsilon}^{0}\right\}$; $E^{0}=\operatorname{tr} \underline{E}^{0}$.

When the last condition of (4) is not to be considered, the residual state of stress only depends on the elastic parameters $E$ and $\nu$, on the shape of the considered domain, and on the incompatibility tensor field $\underline{\underline{E}}^{0}=\underline{\underline{E}}^{0}(x)$. For example when $\underline{E}^{0}$ can be considered as constant in a rock sample, the residual stress field increases as the square of the sample characteristic length: a large sample will split into smaller samples as rocks have a small tensile strength. For a given sample shape, the residual stress field will only depend on the six parameters that define $\underline{\underline{E}}^{0}$.

When $\underline{\underline{E}}^{0}=\underline{\underline{0}}$, there exists a displacement such as $\underline{\underline{\varepsilon}}^{0}=\underline{\varepsilon}^{0}\left\{\underline{\xi}^{0}\right\}$ and residual stresses may vanish to zero; but an additional condition must be met: $\xi^{0}$ must satisfy the last condition of (4).

A simple example (Section 4) is provided by the well known solution of the problem of an hollow cylinder satisfying the Tresca elastoplastic behavior with cohesion $C$. Strains are plane, the external radius $b$ is free of any traction; an increasing pressure $P_{i}$ is applied on the internal radius $a$. The radius $x$ of the elastic-plastic boundary and the plastic strain field are given as a function of $P_{i}$ by (6). It is easy to check that $\underline{\varepsilon}^{0}=\underline{\varepsilon}^{0}\left\{\underline{\xi}^{0}\right\}$, where $\underline{\xi}^{0}$ is given by (7); but, although $\underline{\underline{E}}^{0}=\underline{\underline{E}}\left\{\underline{\varepsilon}^{0}\right\}=\underline{\underline{0}}$, unloading of the tube will not lead to a state in which the stresses are null, as a continuous displacement cannot $\bar{b}$ e found for the whole tube.

Growth of a body is discussed in Section 5. Layers of external radii $b_{1}, b_{2}, \ldots, b_{n}=b$ are successively added to an initial cylinder, radius $a=b_{0}>0$. After a layer has been added, it shrinks according to $\underline{\underline{\varepsilon}}^{\text {shr }}=$ $-\varepsilon\left(\underline{e}_{r} \otimes \underline{e}_{r}+\underline{e}_{\theta} \otimes \underline{e}_{\theta}\right)$. Strains are plane. The radial stress variation induced by the shrinkage of the $\left(b_{i-1}, b_{i}\right)$ layer is given by (8). When $b_{i}-b_{i-1}$ is made very small, a continuous model can be used and the stress build up rate is given by (9); integration leads to (10). It is interesting to notice that any simply connected piece of material cut into the cylinder is stress-free provided it is not crossed by the boundary $r=a$.

\section{Introduction}

Un solide non chargé mécaniquement peut être le siège d'un champ de contraintes non nul, appelé champ de contraintes résiduelles. Ce champ peut avoir deux origines qui ne sont pas exclusives l'une de l'autre : soit le champ de déformation obtenu en annulant le tenseur des contraintes dans la loi de comportement (« déformations initiales ») n'est pas un champ de déformations compatibles; soit on peut considérer (au moins par la pensée) que le corps résulte du «collage» de plusieurs de ses parties qui, considérées individuellement, ne présenteraient pas de contrainte résiduelle; mais qu'il faut déformer pour pouvoir les coller.

\section{Compatibilité, fermeture, discontinuités}

Ce paragraphe vise à préciser certaines définitions et notations. 


\subsection{Conditions de compatibilité}

On note $\underline{\xi}=\underline{\xi}(\underline{x})$ le déplacement d'un point matériel de position $\underline{x}$ dans une configuration de référence. Le tenseur linéarisé des déformations s'écrit par définition :

$$
\underline{\underline{\varepsilon}}\{\underline{\xi}\}=\frac{1}{2}\left(\underline{\underline{\nabla \xi}}+{ }^{\tau} \underline{\underline{\nabla \xi}}\right)
$$

On se donne inversement un tenseur symétrique $\underline{\underline{\varepsilon}}=\underline{\underline{\varepsilon}}(\underline{x})$, fonction suffisamment régulière des coordonnées. Pour trouver un champ de déplacement $\underline{\xi}=\underline{\xi}(\underline{x})$ satisfaisant (1), il faut d'abord que soient vérifiées les conditions de compatibilité, c'est-à-dire que le tenseur symétrique d'incompatibilité $\underline{\underline{E}}=\underline{\underline{E}}\{\underline{\underline{\varepsilon}}\}$ soit nul. Ce tenseur est défini par les deux formules équivalentes :

$$
\begin{aligned}
& \underline{\underline{E}}\{\underline{\underline{\varepsilon}}\}={\underline{\underline{\operatorname{rot}^{d}}}}^{\operatorname{rot}^{g}} \underline{\underline{\varepsilon}} \\
& \underline{\underline{E}}\{\underline{\underline{\varepsilon}}\}=\underline{\underline{\operatorname{grad}}} \operatorname{div} \underline{\underline{\varepsilon}}+{ }^{t} \underline{\underline{\operatorname{grad}}} \operatorname{div} \underline{\underline{\varepsilon}}-\Delta \underline{\underline{\varepsilon}}-\underline{\underline{\operatorname{grad}}} \operatorname{grad}(\operatorname{tr} \underline{\underline{\varepsilon}})-(\operatorname{div} \operatorname{div} \underline{\underline{\varepsilon}}-\Delta \operatorname{tr} \underline{\underline{\varepsilon}}) \underline{\underline{\mathbf{1}}}
\end{aligned}
$$

Si $\underline{\underline{E}}\{\underline{\underline{\varepsilon}}\}=\underline{\underline{0}}$, on dit que $\underline{\underline{\varepsilon}}$ est (géométriquement) compatible. On a toujours div $\underline{\underline{E}}=\underline{0}$; si $\underline{\underline{\varepsilon}}$ est compatible, $\underline{\underline{\underline{E}}}\{\underline{\overline{\underline{\varepsilon}}}\}=\underline{\underline{E}}\left\{\underline{\underline{\underline{\varepsilon^{0}}}}-\underline{\underline{\varepsilon}}\right\}$. Mais, pour satisfaire (1), il faut de plus vérifier les conditions décrites au paragraphe suivant.

\subsection{Condition de fermeture et continuité}

Lorsque $\underline{\underline{\varepsilon}}=\underline{\varepsilon}(\underline{x})$ donné est compatible, on peut calculer le gradient du déplacement puis en faire l'intégration : le déplacement sera défini à une isométrie près. Lorsque le corps considéré n'est pas simplement connexe, il faut qu'il existe un champ de déplacement pour lequel l'isométrie est indépendante du chemin d'intégration retenu (condition de fermeture).

On a supposé implicitement que le champ $\underline{\underline{\varepsilon}}=\underline{\varepsilon}(\underline{x})$ était au moins $C^{2}$ par morceaux. En fait, si $\underline{\underline{\varepsilon}}$ permet de construire un champ de déplacement, ce dernier doit être continu, ce qui implique des conditions nécessaires de régularité pour $\underline{\underline{\varepsilon}}$. Soit $\Gamma$ une surface de discontinuité, de normale $\underline{e}_{3} ;\left(\underline{e}_{1}, \underline{e}_{2}, \underline{e}_{3}\right)$ est une base locale orthonormée; $i$ décrit les indices $1,2,3$ et $\alpha$ décrit les indices 1,2 . Les dérivées $\xi_{i, \alpha}$ doivent être continues, dont aussi $\varepsilon_{\alpha \beta}$; si $\varepsilon_{i j}$ est continu, alors $\varepsilon_{\alpha \beta, i}$ doit être continu, etc. Si de plus $\underline{\underline{\varepsilon}}=\underline{\underline{\varepsilon}}(\underline{x})$ est un champ de déformation candidat à la solution d'un problème de mécanique, il existe une relation de comportement entre $\underline{\underline{\varepsilon}}$ et le tenseur des contraintes; $\underline{\underline{\varepsilon}}$ doit alors satisfaire des conditions supplémentaires résultant de la continuité du vècteur contrainte à travers une surface de discontinuité, qui peut notamment coïncider avec une surface de discontinuité des propriétés mécaniques ou thermiques.

\section{3. État naturel}

\subsection{Détermination des contraintes résiduelles}

Pour calculer les contraintes résiduelles, il faut préciser une relation de comportement. On suppose connu un état mécanique (chargé) du domaine. Les déplacements sont comptés depuis cet état, ou depuis un état qui s'en déduit par une transformation géométrique régulière. On décharge alors le solide en enlevant les forces de volume et les efforts de surface appliqués. Pour fixer les idées, on admet que la décharge s'effectue suivant un comportement élastique, linéaire, homogène, isotrope. On appelle «déformations initiales», soit $\underline{\varepsilon}^{0}=\underline{\underline{\varepsilon}}^{0}(x)$, le champ de déformation obtenu en annulant le tenseur des contraintes dans la relation de comportement :

$$
E \underline{\underline{\varepsilon}}\{\underline{\xi}\}=E \underline{\underline{\varepsilon}}^{0}+(1+v) \underline{\underline{\sigma}}-v(\operatorname{tr} \underline{\underline{\sigma}}) \underline{\underline{1}}
$$


Les contraintes résiduelles (obtenues après déchargement) sont la solution d'un problème qui, en utilisant la définition de $\underline{\underline{\varepsilon}}$ donnée par (3), peut s'écrire :

$$
\begin{array}{ll}
\operatorname{div} \underline{\underline{\sigma}}=\underline{0} & \text { dans } \Omega \\
\underline{\underline{\sigma}} \bullet \underline{n}=\underline{0} & \text { sur } \partial \Omega \\
\underline{\underline{E}}\{\underline{\underline{\varepsilon}}\}=\underline{0} & \text { dans } \Omega \\
{[\underline{\underline{\sigma}}] \bullet \underline{N}=\underline{0}} & \text { sur } \Gamma
\end{array}
$$

Fermeture et continuité de $\underline{\xi}$

On observe que, dès lors que $\underline{E}^{0}=\underline{E}\left\{\underline{\varepsilon}^{0}\right\}$ est connu, l'état chargé n'intervient plus dans le problème de la détermination des contraintes résiduelles que par la dernière condition dans laquelle apparaît la définition retenue de l'état de référence. Les quatre premières équations de (4), avec (3), permettent d'éliminer $\underline{\underline{\varepsilon}}$ par un calcul analogue à celui de Beltrami-Michell [1] pour obtenir le système équivalent :

$$
\begin{aligned}
& \frac{1+v}{E} \Delta \underline{\underline{\sigma}}+\frac{1}{E} \underline{\underline{\operatorname{grad}}} \underline{\underline{\operatorname{grad}}}(\operatorname{tr} \underline{\underline{\sigma}})-\underline{\underline{E}}^{0}+E^{0} \underline{\underline{1}} /(1-v)=0 \quad \text { dans } \Omega \\
& \underline{\underline{\sigma}} \bullet \underline{n}=0 \quad \text { et } \quad \operatorname{div} \underline{\underline{\sigma}}=\underline{0} \quad \operatorname{sur} \partial \Omega, \quad[\underline{\underline{\sigma}}] \bullet N=\underline{0} \quad \text { sur } \Gamma
\end{aligned}
$$

On a noté $E^{0}=\operatorname{tr} \underline{\underline{E}}^{0}$; le calcul utilise la propriété $\operatorname{div} \underline{\underline{E}}^{0}=\underline{0}$. Des équations analogues sont données dans [2].

Hormis la dernière condition de (4), le champ $\overline{\mathrm{de}}$ contraintes résiduelles ne dépend que des paramètres élastiques $E$ et $v$, du domaine considéré et du tenseur d'incompatibilité $\underline{\underline{E}}^{0}$. Dans beaucoup de contextes (mécanique des roches), on ne peut déterminer $\underline{\underline{\varepsilon}}^{0}$, qui résulte d'une histoire impossible à reconstituer; on peut, en revanche, tenter de mesurer $\underline{\underline{E}}^{0}$ ou faire des hypothèses quant à sa forme.

\subsection{Exemple}

Le champ de contraintes résiduelles dans un solide $\Omega$ dépend linéairement du tenseur d'incompatibilité $\underline{\underline{E}}^{0}$, qui a les dimensions de l'inverse du carré d'une longueur. Si $\underline{\underline{E}}^{0}$ est uniforme dans l'espace, non nul, et identique dans deux objets homothétiques, les contraintes résiduelles dans les deux objets sont dans le rapport du carré du rapport d'homothétie; si le matériau a une résistance limitée, un grand objet se casse en plus petits objets (plus vraisemblablement en traction, dans le cas des roches) jusqu'à ce que les contraintes résiduelles n'excèdent plus cette résistance. Tel serait le cas d'un massif rocheux de grandes dimensions dans lequel $\underline{\underline{E}}^{0}$ serait uniforme.

Il existe une taille assez petite en dessous de laquelle un objet présente un tenseur $\underline{E}^{0}$ pratiquement constant.

Si on suppose que c'est le cas pour les éprouvettes de roche prélevées en profondeur et taillées en vue d'essais mécaniques (cylindres d'élancement 2), on peut établir une typologie des distributions de contraintes résiduelles qui dépendent des propriétés élastiques $E$ et $v$ (dans le cas isotrope), de la taille de l'éprouvette, et des six paramètres qui caractérisent $\underline{\underline{E}}^{0}$ [3]. Par exemple, si $E_{r r}=E_{\theta \theta}=v / l^{2}$ et $E_{z z}=-2 / l^{2}$, on a la solution exacte $\sigma_{r r}=E\left(r^{2}-a^{2}\right) / 8 l^{2}, \sigma_{\theta \theta}=E\left(3 r^{2}-a^{2}\right) / 8 l^{2}, \sigma_{z z}=0$ (a est le rayon du cylindre).

\section{3. État naturel}

On dit qu'un domaine $\Omega$ présente un état naturel si les contraintes résiduelles y sont nulles. Un état naturel ne peut exister que si, au moins, le tenseur d'incompatibilité est nul dans tout le domaine. Pour qu'un domaine vérifiant cette propriété présente un état naturel, il faut de plus que la dernière condition de (4) soit satisfaite : si $\underline{\xi}^{0}$ est le champ de déplacement associé au champ de déformations initiales, $\underline{\varepsilon}^{0}=\underline{\varepsilon}^{0}\left\{\underline{\xi}^{0}\right\}, \underline{\xi}^{0}$ doit vérifier les conditions de fermeture et de continuité évoquées à la Section 2.2. Celles-ci sont relatives au domaine considéré 
et à l'état connu du domaine choisi pour définir l'état de référence. On donne des exemples dans les paragraphes suivants.

\section{Cylindre élastoplastique}

On considère un tube cylindrique de rayons $a<b$, réalisé dans un matériau élastoplastique de Tresca, de cohésion $C$. L'état initial est naturel. Les déformations axiales sont bloquées. La contrainte appliquée en $r=b$ est nulle. On fait croître la pression appliquée en $r=a$ depuis zéro jusqu'à $P_{i}=-\sigma_{r r}(a)$. Si $P_{i}$ croît au-delà de la valeur $C\left(1-a^{2} / b^{2}\right)$, apparaissent dans la zone plastique $a<r<x$ des déformations initiales (plastiques), dont le calcul est classique :

$$
\begin{aligned}
& P_{i}=2 C \log \left(\frac{x}{a}\right)+C\left(1-\frac{x^{2}}{b^{2}}\right) \\
& E \underline{\varepsilon}^{0}=2\left(1-v^{2}\right) C\left[\left(\frac{x^{2}}{r^{2}}-1\right)\left(\underline{e}_{\theta} \otimes \underline{e}_{\theta}-\underline{e}_{r} \otimes \underline{e}_{r}\right)\right] \text { pour } a<r<x \\
& E \underline{\underline{\varepsilon}}^{0}=\underline{0} \text { pour } x<r<b
\end{aligned}
$$

Il est facile de vérifier que $\underline{\underline{\varepsilon}}^{0}$ est, dans le domaine $a<r<x$, le champ de déformation du déplacement :

$$
E \underline{\xi}^{0}=2\left(1-v^{2}\right) C\left[\left(r+\frac{x^{2}}{r}\right) \underline{e}_{r}-2 r \theta \underline{e} \theta\right]
$$

On décharge alors la structure; la décharge est élastique si $\log (b / a)<2 / 3$.

(1) On suppose d'abord que l'écoulement libre a été atteint, $x=b$. La décharge ne ramène pas à un état naturel, bien que $\underline{\underline{E}}^{0}=\underline{\underline{0}}$, car le déplacement $\underline{\xi}^{0}$ ne vérifie pas la condition de fermeture. En revanche, si on coupe le tube suivant une ligne $\theta=\theta_{0}$, on le rend simplement connexe et la décharge conduit à un état naturel.

(2) On suppose maintenant $a<x<b$ et on réalise une coupure radiale comme précédemment. La décharge ne conduit plus à un état naturel, car on ne peut prolonger le champ $\underline{\xi}^{0}$ par un champ de déplacement rigide solution de $\underline{\underline{\varepsilon}}^{0}=\underline{\underline{0}}$ dans $x<r<b$ qui soit continu en $r=x$ (comme le laisse prévoir la discontinuité de $\varepsilon_{\theta \theta, r}^{0}$ en $r=x$ ). $\overline{\overline{\mathrm{Il}}}$ faut noter que pour le problème analogue de la sphère creuse, les déformations initiales sont incompatibles.

\section{Un problème de croissance}

Lorsqu'on envisage la croissance d'un objet, décrite par une distribution de vitesse $\underline{\dot{x}}=\dot{x} \underline{n}$ sur la frontière, il faut préciser la loi d'évolution de $\dot{x}$ et les lois physiques qui régissent la croissance. On considère par exemple un cylindre de rayon $a$, soumis à un chargement nul, réalisé dans un matériau vérifiant une loi de comportement élastique, linéaire, homogène, isotrope. L'état initial est naturel; les déformations sont planes.

On fait croître le cylindre en ajoutant successivement des anneaux $b_{i-1} \leqslant r \leqslant b_{i}$, avec $b_{0}=a$ et $b_{n}=b$. Après l'ajout de chaque couche, le matériau ajouté subit un retrait $\underline{\varepsilon}^{\text {ret }}=-\varepsilon\left(\underline{e}_{r} \otimes \underline{e}_{r}+\underline{e}_{\theta} \otimes \underline{e}_{\theta}\right)$ où $\varepsilon>0$. On engendre à chaque ajout, dans le cylindre $r \leqslant b_{i-1}$, une contrainte isotrope uniforme :

$$
2\left(1-v^{2}\right) \delta \sigma_{r r}=2\left(1-v^{2}\right) \delta \sigma_{\theta \theta}=E \varepsilon\left(\frac{b_{i-1}^{2}}{b_{i}^{2}-1}\right)
$$


On peut rendre compte d'une croissance continue en adoptant un modèle dans lequel la couche extérieure $x=x(t)$ croît entre a et $\mathrm{b}$ avec une vitesse $\dot{x}$ qu'on peut supposer constante :

$$
\left(1-v^{2}\right) x \frac{\partial \sigma_{r r}(r, t)}{\partial t}=E \varepsilon \dot{x}, \quad t \geqslant \frac{r-a}{\dot{x}}, \quad \sigma_{r r}\left(r, \frac{r-a}{\dot{x}}\right)=0
$$

On a alors :

$$
\begin{aligned}
& a<r<b, \quad\left(1-v^{2}\right) \sigma_{r r}=E \varepsilon \log \left(\frac{b}{r}\right), \quad\left(1-v^{2}\right) \sigma_{\theta \theta}=E \varepsilon+E \varepsilon \log \left(\frac{b}{r}\right) \\
& 0<r<a, \quad\left(1-v^{2}\right) \sigma_{r r}=\left(1-v^{2}\right) \sigma_{\theta \theta}=E \varepsilon \log \left(\frac{b}{a}\right)
\end{aligned}
$$

On choisit l'état non chargé comme état de référence. De nouveau le tenseur d'incompatibilité est nul, mais on ne peut construire un champ de déplacement $\underline{\xi}^{0}$ continu dans toute la structure, le domaine $a \leqslant r \leqslant b$ étant non simplement connexe et le tenseur $\underline{\underline{\varepsilon}}^{0}$ non continu en $r=a$. En revanche, les contraintes résiduelles disparaissent dans un morceau simplement connexe entièrement contenu soit dans le cylindre initial, soit dans la couronne extérieure ajoutée.

\section{Conclusion}

L'étude met en évidence plusieurs origines possibles pour les contraintes résiduelles dans une structure. Lorsque ces contraintes résultent seulement de l'existence de déformations initiales non compatibles dans la loi de comportement du matériau, l'étude montre le rôle joué par le tenseur d'incompatibilité associé aux déformations initiales : il détermine à lui seul, avec les propriétés élastiques et la forme du corps considéré, la distribution des contraintes résiduelles. Lorsque le tenseur d'incompatibilité est nul dans toute la structure, il faut de plus pouvoir associer au champ de déformations initiales un champ de déplacement continu pour qu'existe un état naturel. Cette possibilité est relative au domaine et à l'état de référence chargé considérés.

\section{Remerciements}

Les auteurs remercient A. Bioget, J.B. Leblond et O. Simionescu pour leurs remarques.

\section{Références}

[1] J. Salençon, Mécanique du Continu, Vol. II, Collection Universités Francophones, Ellipses, Paris, 1995, p. 100.

[2] E. Kröner, Kontinuumstheorie der Versetzungen und Eigenspannungen, Springer-Verlag, 1958.

[3] P. Bérest, G. Vouille, Contraintes résiduelles dans des éprouvettes cylindriques, Rev. Française Géotechn. (2002), soumis. 